\title{
Association of serum levels of vascular endothelial growth factor and thrombospondin-1 to body mass index in polycystic ovary syndrome: a case-control study
}

\author{
Zoya Tahergorabi ${ }^{1}$, Fatemeh Salmani ${ }^{2}$, Samira Hooshmand Jonaidabad ${ }^{3}$, Bahere Behdani ${ }^{3}$, Parisa Yazdi ${ }^{3}$, \\ Mahmoud Zardast ${ }^{4}$, Mitra Moodi ${ }^{5}$

\begin{abstract}
${ }^{1}$ Medical Toxicology and Drug Abuse Research Center (MTDRC), Department of Physiology, School of Medicine, ${ }^{2}$ Social Determinants of Health Research Center, School of Health, ${ }^{3}$ Member of Student Research Committee, School of Medicine, ${ }^{4}$ Cellular and Molecular Research Center, Department of Pathology, School of Medicine, ${ }^{5}$ Social Determinants of Health Research Center, Department of Health Education, School of Health,
\end{abstract} \\ Birjand University of Medical Sciences, Birjand, Iran
}

\section{Purpose}

Polycystic ovary syndrome (PCOS) is a gynecological endocrine disorder that is characterized by disturbances in ovarian blood flow and angiogenesis. The aim of this study was to determine the association of vascular endothelial growth factor (VEGF) and thrombospondin-1 (TSP-1) serum levels with the body mass index (BMI) in patients with PCOS compared with healthy subjects.

\section{Methods}

The study was conducted with 80 subjects in 3 PCOS groups, including normal weight, overweight, and obese PCOS groups, and a control group of healthy subjects $(n=20)$. The participants in all groups completed a questionnaire comprising sociodemographic and obstetric questions. The PCOS diagnosis in the study subjects was confirmed based on the Rotterdam criteria, BMI was determined according to the World Health Organization guidelines, and the lipid accumulation product index was calculated for all groups. Venous blood samples were collected from all participants after fasting to measure the serum levels of fasting blood glucose (FBG), lipids, insulin, VEGF, TSP-1, and leptin.

\section{Results}

Our findings showed that the serum VEGF level was significantly higher in the normal BMI PCOS group than that in the control group $(P=0.03)$, and the TSP-1 level was significantly lower in the obese PCOS group than that in the control group $(P=0.04)$.

\section{Conclusions}

Our study demonstrated that alterations in VEGF and TSP-1 concentrations are dependent on BMI. Because abnormal ovarian angiogenesis is considered to be the main feature of PCOS, the study of ovarian angiogenic imbalance is proposed as a new tool for PCOS diagnosis and management.

Keywords: Body mass index; Polycystic ovarian syndrome; Thrombospondin-1; Vascular endothelial growth factor

\section{Introduction}

Polycystic ovary syndrome (PCOS) is a common gynecological endocrine disorder that occurs during a woman's reproductive years. It is characterized by a wide spectrum of endocrine and metabolic disorders, including obesity, hyperandrogenism, insulin resistance, and loss of the intraovarian autoregulatory mechanism, resulting in anovulation, dyslipidemia, and
Received: 2019.02.22. Revised: 2019.04.15. Accepted: 2019.06.16. Corresponding author: Mitra Moodi

Social Determinants of Health Research Center, Department of Health Education, School of Health, Birjand University of Medical Sciences, Birjand, Iran

E-mail: mitra_m2561@yahoo.com

https://orcid.org/0000-0002-8877-6609

Articles published in Obstet Gynecol Sci are open-access, distributed under the terms of the Creative Commons Attribution Non-Commercial License (http://creativecommons. org/licenses/by-nc/3.0/) which permits unrestricted non-commercial use, distribution, and reproduction in any medium, provided the original work is properly cited.

Copyright $\odot 2019$ Korean Society of Obstetrics and Gynecology 


\section{Obstetrics \& Gynecology Science}

Zoya Tahergorabi, et al. Association VEGF \& TSP-1 to BMI in PCO patients

hyperglycemia [1,2]. Although a high percentage of women with PCOS are obese, $40-50 \%$ of women with PCOS are not obese. Thus, obesity per se is not necessarily intrinsic to PCOS, as insulin resistance is present in lean patients with PCOS as well as in obese ones $[3,4]$.

PCOS, as many other complex syndromes, does not have a single diagnostic marker to provide a gold standard for reference. Consequently, several sets of criteria have been proposed for its diagnosis. However, the disparity between the diagnosis guidelines, although minor, has been associated with a variation in the diagnosis and treatment of PCOS. For example, the Rotterdam criteria were developed based on expert opinions and are valuable both clinically and scientifically. In 2012, the PCOS Consensus Workshop Group at its third meeting reported that the diagnosis of PCOS in adolescents is similar to that in adults and should thus meet the Rotterdam criteria $[5,6]$. PCOS can be diagnosed by the presence of 2 of the following 3 features: 1) oligo- or anovulation, 2) clinical and/or biochemical signs of hyperandrogenism, and 3) polycystic ovaries on ultrasonography [7].

The coordinated interaction between pro- and antiangiogenic factors within the ovary creates a complex process of angiogenesis that regulates important physiological processes, such as ovarian folliculogenesis, ovulation, and formation of the corpus luteum, which require angiogenesis. Angiogenesis is defined as the formation of new blood vessels from preexisting vasculature and as such, it is activated in the wound healing process and is also involved in the female reproductive system during non-pregnant menstrual cycles and during the implantation of embryos in pregnancy [8].

The pathogenesis of PCOS is not well understood; however, accumulating evidence suggests that a disturbance in the ovarian blood flow and angiogenesis leads to disorders of ovulation and subfertility in women with PCOS [9].

Angiogenic and angiostatic factors, such as the vascular endothelial growth factor (VEGF) family, angiopoietin, basic fibroblast growth factor (bFGF), transforming growth factor $\beta$ (TGF $\beta)$, matrix metalloproteinase, platelet-derived growth factor (PDGF), and thrombospondin (TSP-1) are coordinately expressed during the ovarian cycle [10].

Human VEGF, the most prominent and potent angiogenic factor in cyclic angiogenesis and vascular permeability, stimulates endothelial proliferation, migration, and survival [10]. Several studies have demonstrated that increased ovarian vascularity correlates with increased serum VEGF levels in women with PCOS $[11,12]$. In a study by Loebig et al. [13] in 2010, the authors demonstrated a positive correlation between the plasma VEGF concentration and a large body mass index (BMI) range between $17.6 \mathrm{~kg} / \mathrm{m}^{2}$ and $43.6 \mathrm{~kg} / \mathrm{m}^{2}$ in healthy male subjects.

TSP-1, originally isolated from platelets and megakaryocytes, can inhibit follicular angiogenesis and induce apoptosis in granulosa cells [13]. It has been shown that a reduced expression of TSP-1 can lead to aggressive angiogenesis [14]. In a 2008 study by Varma et al. [15] on TSP-1 gene expression in the subcutaneous adipose tissue, TSP-1 had a positive association with the BMI in 86 nondiabetic subjects across a wide range of $\mathrm{BMI}$. This result is in contrast to that of a 2009 investigation by Tan et al. [16] where 19 women with PCOS and 54 women in a control group were recruited from an infertility clinic. In that study, 5 subjects with PCOS and 7 subjects in the control group withdrew before the study could be completed, but there were no significant associations between the circulating TSP-1 and the TSP-1 levels in the adipose tissue with the BMI $[15,16]$. It can thus be concluded that TSP-1 might have bidirectional effects.

To the best of our knowledge, there are few studies on the relationship between angiogenic and angiostatic factors and $\mathrm{BMI}$ in PCOS. Therefore, the current study aimed to determine the association of VEGF and TSP-1 serum levels with the BMI in patients with PCOS compared with healthy subjects.

\section{Materials and methods}

The present case-control study was conducted in the Department of Physiology in collaboration with the Department of Biochemistry and Obstetrics and Gynecology at Birjand University of Medical Sciences. The research protocol was approved by the Institutional Review Board of the research committee of Birjand University of Medical Sciences, and the study was performed in accordance with the approved guidelines.

The subjects in the study were 80 women, aged 15-45 years, from the South Khorasan province of Birjand. The sample size was estimated to be 19.3 persons based on the formula for comparing 2 means, and the results of the Maxwell study and the homeostasis model assessment (HOMA) index with $\mathrm{S} 1=0.6, \mathrm{~S} 2=1.8, d=1.4, a=0.05$ and $\beta=0.1$, in 


\section{Obstetrics \& Gynecology Science}

Vol. 62, No. 6, 2019

which 20 women in each group were considered [17].

Among the 80 subjects, there were 60 women with PCOS, including 20 women of normal weight based on the BMI (18.5<BMl<24.9), 20 overweight women (25>BMl>29.9), and 20 obese women (BMI $\geq 30)$, and 20 healthy women (control group). The control group was selected randomly from healthy females who were referred to the Birjand Health Lab Center for premarital tests, environmental, and occupational healthcare. Case and control groups were matched for age. None of the patients with PCOS were taking drugs for at least 6 months prior to the study.

A history of other causes of hyperandrogenism, such as congenital adrenal hyperplasia, androgen secreting tumor, Cushing's syndrome, and other insulin resistance conditions (acromegaly), hypothyroidism, hyperprolactinemia, pregnancy, menopause, and use of medications resulted in withdrawal of individuals from the study.

Participants in all groups completed the study questionnaire after consent was provided according to the criteria of the Ethics Committee of the Faculty of Medicine of the Birjand University of Medical Sciences. The sociodemographic section of the 2-part questionnaire included questions about age, education level, spouse's education level, marriage age, first gestational age, number of children, and smoking status. The obstetric questions pertained to gravidity, parity, abortion(s), number of live births, first menstrual age, regularity of menstrual bleeding, contraception method, usage of assisted reproductive treatment, and duration of PCOS.

PCOS diagnosis was confirmed in the case groups according to the Rotterdam criteria, including oligo- or anovulatory menstrual cycle, defined as a menstrual period lasting more than 40 days or fewer than 9 cycles per year. Clinical or laboratory hyperandrogenism would include hirsutism, the growth of terminal hair above the upper lip and on the chin/ face, chest, back, abdomen, arms, and thighs based on the Ferriman-Gallwey scoring system, where hirsutism is defined as a score above 8 or increased levels of testosterone and/or dehydroepiandrosterone [18]. Abdominal sonography was performed during the first 5 days of the normal menstrual cycle. A sonogram result for polycystic ovaries according to the Rotterdam criteria is considered to be 12 or more follicles in each ovary with a diameter of 2 to $9 \mathrm{~mm}$ [19].

\section{Anthropometric measurements}

The weight and height of all case and control group partici- pants were measured. The participants were weighed on a calibrated scale while wearing light clothes and no shoes (Seca, Hamburg, Germany) and height without shoes was measured using a stadiometer with a fixed vertical backboard and an adjustable headpiece. BMI was calculated by taking the weight in $\mathrm{kg}$ divided by the square of the height in meters and then graded according to the World Health Organization (WHO) classification [20]. The lipid accumulation product (LAP) index was calculated based on the following formula: (waist circumference [cm]) - 58)xfasting concentration of circulation triglycerides [21]. Insulin resistance in the patients with PCOS was based on a cutoff point of 34.5 [22].

\section{Sample collection}

Following an overnight fast (12 hours), participants' blood samples were taken from the left brachial vein under aseptic conditions for measurement of the serum levels of FBS, lowdensity lipoprotein cholesterol (LDL-C), high-density lipoprotein cholesterol (HDL-C), triglycerides, insulin (type 6000, Cobas, Hitachi, Tokyo, Japan), VEGF (human VEGF ELISA Kit, Hangzhou EastbiopharmCo., Ltd, USA), TSP-1 (human TSP-1 ELISA Kit, Hangzhou EastbiopharmCo., Ltd), and leptin (human leptin ELISA Kit, Hangzhou EastbiopharmCo., Ltd).

\section{Statistical analysis}

Data were reported as mean \pm standard deviation. The normal distribution of the data was assessed using the KolmogorovSmirnov test. One-way analysis of variance (ANOVA), Kruskal-Wallis, Pearson, and Spearman correlation tests were conducted for comparison of the variables among the PCOS groups and the control group. Secondary analyses were logistic regression models. A model was applied to compute the probability for PCOS by controlling for potential confounders and estimating the odds ratio. We used principal component analysis and the forward method for selection of the best regression analysis. In addition, the receiver operating characteristic (ROC) curve was applied for determining the power of prediction of the proposed model. SPSS Version 16 Statistical Package (IBM Corporation, Armonk, NY, USA) and Excel 2010 (Microsoft Co. Redmond, WA, USA) were used for the statistical analysis. A $P$-value $<0.05$ was considered statistically significant for all statistical tests. 


\section{Obstetrics \& Gynecology Science}

Zoya Tahergorabi, et al. Association VEGF \& TSP-1 to BMI in PCO patients

\section{Results}

Our results consisted of three sections: Section 1 includes descriptive statistics and one-way ANOVA results for comparing the means among the 4 groups. In section 2, logistic regression results are presented, and section 3 includes the final model for making predictions about PCOS.

\section{Descriptive statistics and one-way analysis of variance}

The study included 80 women participants in 4 groups: normal, overweight, and obese BMI patients with PCOS and a control group. The groups were matched in terms of age $(P=0.68)$, occupation $(P=0.05)$, residence $(P=0.06)$, and income status $(P=0.4)$. Table 1 shows a demographic, anthropometric, biochemical, and hormonal profiles of the cases and the control group. The Bonferroni post hoc test showed that serum levels of FBS and triglycerides were significantly higher in the obese PCOS group than in the normal BMI PCOS group $(P=0.04, P \leq 0.001)$ and the control group
$(P=0.03, P \leq 0.001)$. The total cholesterol $(P=0.04)$ and LDL$C(P=0.03)$ levels in the obese PCOS group were significantly higher than those of the control group, and HDL-C was significantly lower in the obese PCOS group than in the normal BMI PCOS ( $P=0.04)$ and control groups $(P=0.01)$.

Homeostatic model assessment for insulin resistance (HOMA-IR) was significantly higher in the obese PCOS group than in the normal BMI PCOS $(P=0.001)$ and the control group ( $P=0.002$ ). Thirty-one percent of the overweight patients with PCOS and $53 \%$ of the obese patients with PCOS had insulin resistance, whereas this trait was only present in $5 \%$ of the patients of the normal $\mathrm{BMI}$ and control groups (HOMA-IR $\geq 3.8$ ).

The plasma VEGF level was significantly higher in the normal BMI PCOS group than in the control group (Fig. 1), and TSP-1 was significantly lower in the obese PCOS group than in the control group (Fig. 2). The correlation between HOMA$I R$ and the LAP-index is shown in Figure $A$ in the Supplementary Data 1 . The LAP-index was positively correlated with HOMA-IR $(r=0.73, P \leq 0.001)$.

Table 1. Comparison of the demographic, biochemical, and hormonal profile of the study participants in the four groups

\begin{tabular}{|c|c|c|c|c|c|}
\hline Variables & Normal BMI & Overweight & Obese & Control & $P$-value \\
\hline Age (yr) & $25.1 \pm 4.26$ & $25.68 \pm 5.47$ & $26.05 \pm 8.22$ & $24.5 \pm 5.72$ & 0.86 \\
\hline BMI $\left(\mathrm{kg} / \mathrm{m}^{2}\right)$ & $21.31 \pm 2.22$ & $26.94 \pm 1.46$ & $34.40 \pm 2.75$ & $21.07 \pm 3.16$ & $\leq 0.001^{b)}$ \\
\hline WC $(\mathrm{cm})$ & $73.42 \pm 6.16$ & $86.68 \pm 6.91$ & $100.76 \pm 7.62$ & $74.95 \pm 6.37$ & $\leq 0.001^{\text {b) }}$ \\
\hline $\mathrm{SBP}(\mathrm{mmHg})$ & $106.05 \pm 10.6$ & $105.50 \pm 4.78$ & $117.86 \pm 5.46$ & $98.70 \pm 7.31$ & $0.03^{b)}$ \\
\hline $\mathrm{DBP}(\mathrm{mmHg})$ & $70.52 \pm 10.12$ & $71.25 \pm 7.41$ & $75.26 \pm 13.89$ & $69.25 \pm 5.44$ & 0.26 \\
\hline FBS (mg/dL) & $90.65 \pm 5.98$ & $91.31 \pm 5.35$ & $97.47 \pm 11.76$ & $90.25 \pm 6.16$ & $0.01^{\mathrm{b})}$ \\
\hline $\mathrm{TC}(\mathrm{mmol} / \mathrm{L})$ & $161 \pm 41.44$ & $177.06 \pm 36.06$ & $187.78 \pm 37.27$ & $156.80 \pm 25.39$ & $0.03^{b)}$ \\
\hline TG (mmol/L) & $76.05 \pm 38.06$ & $113.81 \pm 56.84$ & $149.05 \pm 61.47$ & $75.35 \pm 39.07$ & $\leq 0.001^{b)}$ \\
\hline $\mathrm{HDL}-\mathrm{C}(\mathrm{mmol} / \mathrm{L})$ & $48.30 \pm 10.42$ & $42.80 \pm 7.83$ & $39.78 \pm 8.36$ & $49.15 \pm 10.71$ & $0.009^{b)}$ \\
\hline LDL-C (mmol/L) & $89.15 \pm 33.37$ & $109.33 \pm 32.34$ & $112.63 \pm 30.88$ & $86.25 \pm 17.44$ & $0.01^{b)}$ \\
\hline Insulin & $9.03 \pm 3.95$ & $13.62 \pm 4.81$ & $22.98 \pm 18.44$ & $9.33 \pm 3.63$ & $\leq 0.001^{b)}$ \\
\hline HOMA-IR & $2.03 \pm 0.84$ & $3.08 \pm 1.16$ & $5.75 \pm 1.31$ & $2.08 \pm 0.87$ & $\leq 0.001^{b)}$ \\
\hline LAP-index ${ }^{a}$ & $57.69(28.25-94.16)$ & 153.79 (99.78-216.07) & $320.11(241.25-451.77)$ & 57.38 (39.79-106.06) & $\leq 0.001^{b)}$ \\
\hline VEGF $^{\text {a) }}$ & 384.15 (253.72-1844.57) & $248.8(163.12-404.57)$ & $353.2(258.8-813.4)$ & $412.05(287.5-504.85)$ & $0.03^{b)}$ \\
\hline Leptin ${ }^{\text {a) }}$ & $0.77(0.59-4.22)$ & $0.66(0.53-5.43)$ & $0.76(0.45-2.01)$ & $1.01(0.53-6.15)$ & 0.42 \\
\hline TSP-1 ${ }^{\text {a) }}$ & $45.85(31.25-218.57)$ & 33.25 (27.92-198.65) & $38.7(28.2-132.2)$ & $82.74(43.9-527.7)$ & $0.04^{\mathrm{b})}$ \\
\hline
\end{tabular}

Values are presented as mean \pm standard deviation (ANOVA test).

BMI, body mass index; WC, waist circumference; SBP, systolic blood pressure; DBP, diastolic blood pressure; FBS, fasting blood sugar; TC, total cholesterol; TG, triglyceride; HDL-C, high-density lipoprotein cholesterol; LDL-C, low density lipoprotein cholesterol; HOMA, homeostasis model assessment; LAP, lipid accumulation product; VEGF, vascular endothelial growth factor; TSP-1, thrombospondin-1.

a) Median and 25-75 inter-quartile range (Kruskal-Wallis test); ${ }^{b} P<0.05$ is significant. 


\section{Obstetrics \& Gynecology Science}

Vol. 62, No. 6, 2019

\section{Prediction of polycystic ovary syndrome with a logistic regression model}

The results in this section consider the logistic regression model. Table 1 shows the significant difference between the variables. These variables should be controlled in the logistic regression model. The final model was chosen by principal component analysis (PCA) and the forward method (Section A, Supplementary Data 1). The selected model is as follows: logistic $(P C O S)=\beta_{0}+\beta_{1} B M 1+\beta_{2}$ TSP $1+\beta_{3}$ VEGF + $\beta_{4}$ Leptin $\times$ TSP 1

The estimated parameters are presented in Table 2. We used an ROC curve to determine an accurate detection of PCOS status (PCOS positive, PCOS negative). Finally, sensitivity, specificity, true positive, true negative, false positive, and false negative were computed; the ROC curve is shown in Fig. 3. According to the ROC curve result, the best cutoff point was 0.66 with a sensitivity of 0.74 and a specificity of 0.94 . The area under the ROC curve (AUC) was 0.93 with a confidence interval of 0.88-0.97 $(P<0.001)$. For more details, refer to the Supplementary Data 1.

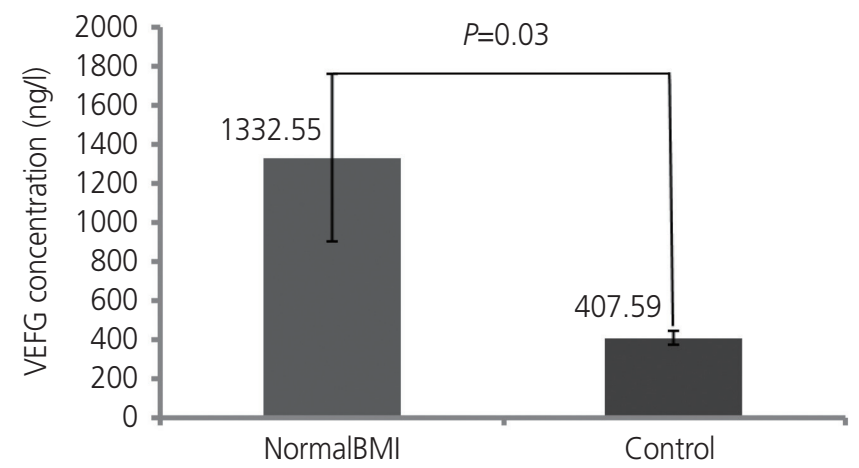

Fig. 1. Comparison of the plasma VEGF levels in the normal BMI PCOS group and the control group. VEGF, vascular endothelial growth factor; BMI, body mass index; PCOS, polycystic ovary syndrome.

\section{Discussion}

Our results revealed a significant increase in the serum VEGF level and a significant decrease in the serum TSP-1 level in a dependent manner to BMI in women with PCOS.

The significantly higher VEGF levels that we found in the patients of the normal BMI PCOS group, but not in those of the overweight and obese PCOS groups, are in line with the results of a study by Loebig et al. [13] in 2010 on 15 normalweight, 15 low-weight, and 15 obese male subjects aged 18-30 years. These results indicate that body composition does not influence angiogenesis through the systemic level of VEGF.

VEGF is an endothelial cell mitogen and a heparin-binding glycoprotein that acts as a main factor during physiological and pathological angiogenesis [10]. One possible explanation is the accumulating evidence indicating that PCOS is associated with chronic inflammation, which, in turn, can lead to an abnormal angiogenesis in the ovary tissue [23]. In addition, the increased VEGF level in the serum of patients with PCOS could be caused by an increased number of actively

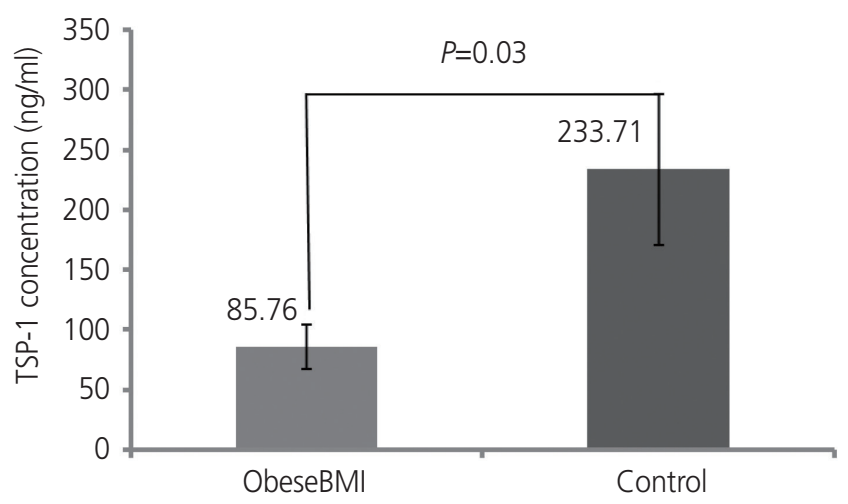

Fig. 2. Comparison of the plasma TSP-1 levels in the obese BMI PCOS group and the control group. TSP-1, thrombospondin-1; BMI, body mass index; PCOS, polycystic ovary syndrome.

Table 2. Estimated parameters and odd ratio for a final model and collinearity index (VIF)

\begin{tabular}{lccccccc}
\hline Variables & B & SE & Wald & df & Sig. & Exp(B) & VIF \\
\hline BMI & 0.455 & 0.129 & 12.331 & 1 & 0.000 & 1.576 & 2.63 \\
TSP-1 & -0.100 & 0.040 & 6.405 & 1 & 0.011 & 0.905 & 6.45 \\
VEGF & 0.004 & 0.002 & 4.660 & 1 & 0.031 & 1.004 & 1.45 \\
Leptin by TSP-1 & 0.014 & 0.006 & 6.060 & 1 & 0.014 & 1.014 & \\
Constant & -6.752 & 2.329 & 8.405 & 1 & 0.004 & 0.001 & \\
\hline
\end{tabular}

$\mathrm{SE}$, standard error; df, degrees of freedom; BMI, body mass index; VEGF, vascular endothelial growth factor; TSP-1, thrombospondin-1. 


\section{Obstetrics \& Gynecology Science}

Zoya Tahergorabi, et al. Association VEGF \& TSP-1 to BMI in PCO patients

secreting granulosa lutein cells and an upregulated gene expression of these cells, due to the fact that granulosa lutein cells are the most important source of VEGF in the female reproductive system [24]. In the recent years, several compounds capable of inhibiting the VEGF properties have been developed as a potential therapy for treating cancers and other disorders involving angiogenic alterations.

The significant decrease of TSP-1 levels in a dependent manner to $\mathrm{BMI}$ in our study is in accordance with the findings of Tan et al. [16] in 2009, where 19 patients with PCOS and 54 control subjects were recruited from an infertility clinic. However, our results are inconsistent with those of Varma et al. [15], who evaluated 86 subjects (70 women and 16 men; aged 21-66 years) in 2008.

TSP-1 is a large glycoprotein that is involved in the regulation of matrix structures, wound healing, and tumorigenesis [24]. Evidence demonstrated that the TSP-1 serum level is not related to the BMI, in contrast to a study that found increased TSP-1 serum levels in obese patients with PCOS who had insulin resistance [25].

In the present study, it is possible that the low TSP-1 serum level was negatively associated with the changes in glucose and triglyceride levels.

Tada et al. [26] showed that high glucose levels, via protein kinase $C$ pathway activation and TSP-1 synthesis in the mesangial cells, promoting TGF $\beta 1$ activation, led to an increased consumption of TSP-1, resulting in increased angiogenesis in patients with PCOS. TSP-1 directly or indirectly affects the insulin resistance and hyperinsulinemia and is associated with the altered expression of lipoprotein lipase and hepatic lipase $[25,27]$. Consequently, TSP-1 can induce dyslipidemia in patients with PCOS, as in our study the insulin concentration was significantly higher in the obese and overweight patients with PCOS than in the healthy subjects.

In addition, the increase in the size of adipocytes led to a relatively decreased blood perfusion to each adipocyte and resultant tissue hypoxia. Adipose tissue hypoxia has consistently been reported in various animal models of obesity, including genetically obese ob/ob and $\mathrm{db} / \mathrm{db}$ mice, obese Zucker rats, and high-fat diet (HFD)-fed mice. In response to hypoxia, hypoxia-inducible factor-1 alpha (HIF-1a) expression and hypoxia-targeted gene expression are increased in white adipocytes, in which VEGF is considered to be a direct transcriptional target of HIF-1a that acts as an angiogenic driving force for rapidly expanding the white adipocyte tissues (WAT).

Adipose tissue hypoxia in obesity is not only the primary driving force of angiogenesis, but can also initiate chronic inflammation. Chronic inflammation in WAT is exhibited by infiltrating macrophages, neutrophils, and immune cells, which, together with adipocytes and stromal vascular cells, produce various inflammatory cytokines. Inflammatory cells, in turn, release angiogenic factors, including VEGF, angio-
A

ROC Curve / 0.660272519857459 /

$\mathrm{AUC}=0.925$

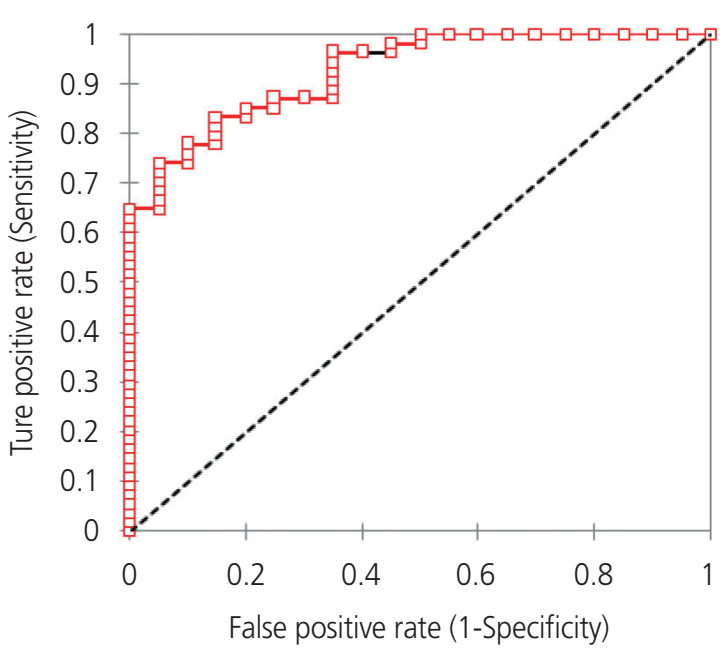

B

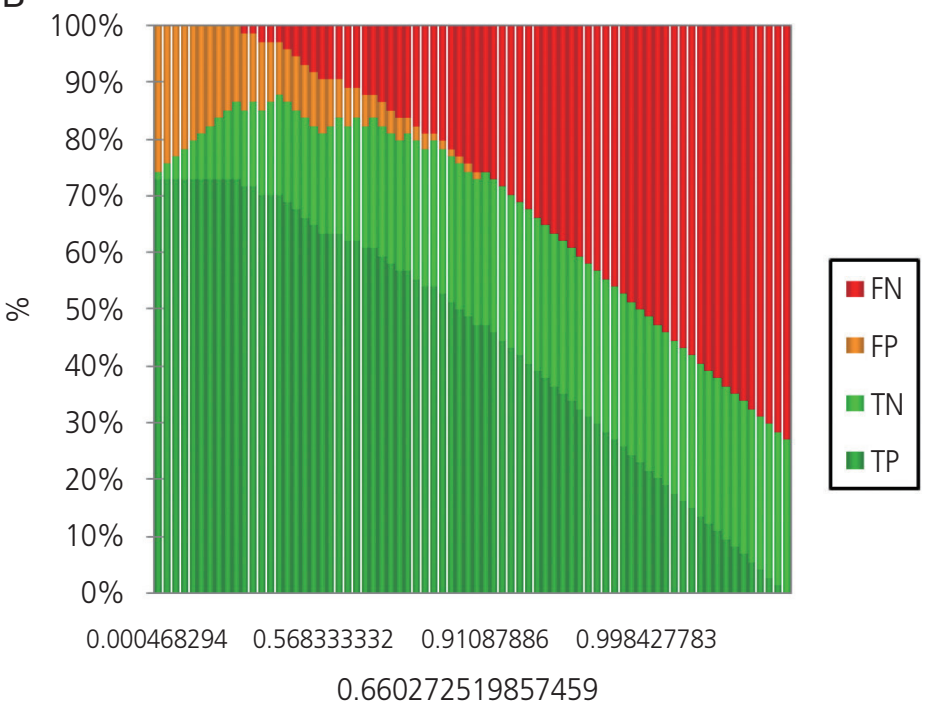

Fig. 3. The diagnostic values of logistic model for patients with polycystic ovary syndrome (PCOS) and control group. (A) Receiver operating characteristic (ROC) curve for PCOS and control group with final model. (B) True positive (TP), true negative (TN), false positive (FP), false negative (FN) for PCOS and control group. AUC, area under the curve. 


\section{Obstetrics \& Gynecology Science}

Vol. 62, No. 6, 2019

poietins, bFGF, hepatocyte growth factor (HGF), PDGF, and transforming growth factor (TGF-B), which have mitogenic and migratory effects on the endothelium. Thus, angiogenesis maintains inflammation by supplying oxygen and nutrients to the cells in the inflamed area $[28,29]$.

Compelling evidence supports the crucial role of adipose tissue angiogenesis in the modulation of insulin sensitivity. In a study that included TNP-470-treated (a generic angiogenesis inhibitor) obese mice, the reduction in adipose vascular density was correlated with an improved insulin sensitivity. Several studies on the vascular function in the modulation of insulin sensitivity focused primarily on regulating the VEGF production in the pancreatic islets and VEGF sensitivity in peripheral tissues, such as adipose and muscle tissues. In parallel, in recent years, targeting angiogenesis for the treatment of obesity and its related metabolic disorders is an exciting therapeutic approach that may prove to be beneficial for millions of obese patients and those with diabetes [30-32].

The logistic regression model was further examined as a model for the prediction of PCOS. To achieve this, a ROC analysis was performed (Fig. 3A). With an AUC of 0.925 (95\% $\mathrm{Cl}, 0.876-0.974)$ and a cutoff level of 0.66 , the results demonstrated that the final model is a good predictive model for PCOS. The sensitivity and specificity of the model were 0.74 and 0.94 , respectively. It was also observed that BMI remained associated with PCOS even after adjusting for other potentially confounding variables (including TSP-1, VEGF, and the interaction between TSP-1 and leptin).

In the present study, it was noted that the LAP index had a high positive correlation with the HOMA index in patients with PCOS.

An LAP index based on waist circumference and triglyceride levels is an ordinal, safe, and inexpensive screening tool for insulin resistance and other possible comorbidities related to this metabolic disorder, such as cardiovascular diseases, in patients with PCOS. This is in agreement with the findings of previous studies that have shown that the LAP index might be more accurate than the HOMA index for the diagnosis of insulin resistance in metabolic disorders [22,33].

The present study is not without limitations. For example, our sample size was relatively small due to the low population density of the geographic area in which the study was conducted. Consequently, we were unable to match the case groups with the control group in terms of BMI. In addition, a metformin group was not included in the study.
Nonetheless, the study has a number of strengths. For example, we were able to recruit 60 women with a newly diagnosed PCOS along with 20 healthy age-matched controls. Moreover, our findings relate to the $3 \mathrm{WHO}$ classification criteria for normal, overweight, and obese women with PCOS according to the BMI.

In conclusion, the results of this study showed that the increased VEGF and decreased TSP-1 levels in women with PCOS are associated with the BMI. The interactions between the angiogenic and angiostatic factors are very complex, and BMI may thus be a relevant factor in this context. In addition, the present study allowed the construction of a model with a relatively high predictive power for the prediction of positive or negative probability for PCOS, which could be useful in clinical practice.

\section{Acknowledgments}

The authors would like to thank the Vice Chancellor for Research and Technology of the Birjand University of Medical Sciences for funding this study (Research Project No. 4495). In addition, the authors would like to thank Dr. Marzieh Ataee, a gynecologist and obstetrician.

\section{Conflict of interest}

The authors have no conflicts of interest to declare related to this study.

\section{Ethical approval}

This study was approved by the University Ethics Committee (approval number IR.bums.REC.1396.69). All procedures were performed in accordance with the Declaration of Helsinki and the criteria of the ethics committee of the Faculty of Medicine of the Birjand University of Medical Sciences.

\section{Patient consent}

Informed consent was obtained from all participants. 


\section{Obstetrics \& Gynecology Science}

Zoya Tahergorabi, et al. Association VEGF \& TSP-1 to BMI in PCO patients

\section{Supplementary material}

Supplementary Data 1 associated with this article can be found online at https://doi.org/10.5468/ogs.2019.62.6.420.

\section{References}

1. Qu J, Che Y, Xu P, Xia Y, Wu X, Wang Y. The higher response of vascular endothelial growth factor and angiotensin-ii to human chorionic gonadotropin in women with polycystic ovary syndrome. Int J Fertil Steril 2015;8:373-8.

2. Peitsidis P, Agrawal R. Role of vascular endothelial growth factor in women with PCO and PCOS: a systematic review. Reprod Biomed Online 2010;20:444-52.

3. Diamanti-Kandarakis E. Role of obesity and adiposity in polycystic ovary syndrome. Int J Obes 2007;31 Suppl 2:58-13.

4. Jin $\mathrm{CH}$, Yuk JS, Choi KM, Yi KW, Kim T, Hur JY, et al. Body fat distribution and its associated factors in Korean women with polycystic ovary syndrome. J Obstet Gynaecol Res 2015;41:1577-83.

5. El Hayek S, Bitar L, Hamdar LH, Mirza FG, Daoud G. Poly cystic ovarian syndrome: an updated overview. Front Physiol 2016;7:124.

6. Wang R, Mol BW. The Rotterdam criteria for polycystic ovary syndrome: evidence-based criteria? Hum Reprod 2017;32:261-4.

7. Bani Mohammad M, Majdi Seghinsara A. Polycystic ovary syndrome (PCOS), diagnostic criteria, and AMH. Asian Pac J Cancer Prev 2017;18:17-21.

8. Osz K, Ross M, Petrik J. The thrombospondin-1 receptor CD36 is an important mediator of ovarian angiogenesis and folliculogenesis. Reprod Biol Endocrinol 2014;12:21.

9. Geva E, Jaffe RB. Role of vascular endothelial growth factor in ovarian physiology and pathology. Fertil Steril 2000;74:429-38.

10. Holmes DI, Zachary I. The vascular endothelial growth factor (VEGF) family: angiogenic factors in health and disease. Genome Biol 2005;6:209.

11. Greenaway J, Connor K, Pedersen HG, Coomber BL, LaMarre J, Petrik J. Vascular endothelial growth factor and its receptor, Flk-1/KDR, are cytoprotective in the extravascular compartment of the ovarian follicle. Endocrinol- ogy 2004;145:2896-905.

12. Ferrara N. The role of VEGF in the regulation of physiological and pathological angiogenesis. EXS 2005;(94):209-31.

13. Loebig M, Klement J, Schmoller A, Betz S, Heuck N, Schweiger $U$, et al. Evidence for a relationship between VEGF and BMI independent of insulin sensitivity by glucose clamp procedure in a homogenous group healthy young men. PLoS One 2010;5:e12610.

14. Lawler J, Detmar M. Tumor progression: the effects of thrombospondin-1 and -2. Int J Biochem Cell Biol 2004;36:1038-45.

15. Varma V, Yao-Borengasser A, Bodles AM, Rasouli N, Phanavanh B, Nolen GT, et al. Thrombospondin-1 is an adipokine associated with obesity, adipose inflammation, and insulin resistance. Diabetes 2008;57:432-9.

16. Tan BK, Adya R, Chen J, Farhatullah S, Heutling D, Mitchell $D$, et al. Metformin decreases angiogenesis via NF-kappaB and Erk1/2/Erk5 pathways by increasing the antiangiogenic thrombospondin-1. Cardiovasc Res 2009;83:566-74.

17. Maxel T, Svendsen PF, Smidt K, Lauridsen JK, Brock B, Pedersen SB, et al. Expression patterns and correlations with metabolic markers of zinc transporters ZIP14 and ZNT1 in obesity and polycystic ovary syndrome. Front Endocrinol (Lausanne) 2017;8:38.

18. Sanchez N. A life course perspective on polycystic ovary syndrome. Int J Womens Health 2014;6:115-22.

19. F. BCJM. Revised 2003 consensus on diagnostic criteria and long-term health risks related to polycystic ovary syndrome: Rotterdam ESHRE/ASRM-Sponsored 3rd PCOS Consensus Workshop Group. Fertil Steril 2004;81:1925.

20. Souza Dos Santos AC, Soares NP, Costa EC, de Sá JC, Azevedo GD, Lemos TM. The impact of body mass on inflammatory markers and insulin resistance in polycystic ovary syndrome. Gynecol Endocrinol 2015;31:225-8.

21. Kahn HS. The "lipid accumulation product" performs better than the body mass index for recognizing cardiovascular risk: a population-based comparison. BMC Cardiovasc Disord 2005;5:26.

22. Wiltgen D, Benedetto IG, Mastella LS, Spritzer PM. Lipid accumulation product index: a reliable marker of cardiovascular risk in polycystic ovary syndrome. Hum Reprod 2009;24:1726-31. 


\section{Obstetrics \& Gynecology Science}

Vol. 62, No. 6, 2019

23. Duleba AJ, Dokras A. Is PCOS an inflammatory process? Fertil Steril 2012;97:7-12.

24. Harada M, Peegel H, Menon KM. Expression of vascular endothelial growth factor A during ligand-induced down-regulation of luteinizing hormone receptor in the ovary. Mol Cell Endocrinol 2010;328:28-33.

25. Bhattacharyya S, Marinic TE, Krukovets I, Hoppe G, Stenina OI. Cell type-specific post-transcriptional regulation of production of the potent antiangiogenic and proatherogenic protein thrombospondin-1 by high glucose. J Biol Chem 2008;283:5699-707.

26. Tada H, Kuboki K, Nomura K, Inokuchi T. High glucose levels enhance TGF-beta1-thrombospondin-1 pathway in cultured human mesangial cells via mechanisms dependent on glucose-induced PKC activation. J Diabetes Complications 2001;15:193-7.

27. Ebrahimi-Mamaghani M, Saghafi-Asl M, Pirouzpanah S, Aliasgharzadeh A, Aliashrafi S, Rezayi N, et al. Association of insulin resistance with lipid profile, metabolic syndrome, and hormonal aberrations in overweight or obese women with polycystic ovary syndrome. J Health Popul Nutr 2015;33:157-67.

28. Tahergorabi Z, Khazaei M. The relationship between inflammatory markers, angiogenesis, and obesity. ARYA Atheroscler 2013;9:247-53.

29. Goossens GH, Blaak EE. Adipose tissue oxygen tension: implications for chronic metabolic and inflammatory diseases. Curr Opin Clin Nutr Metab Care 2012;15:539-46.

30. Sun K, Halberg N, Khan M, Magalang UJ, Scherer $\mathrm{PE}$. Selective inhibition of hypoxia-inducible factor $1 \mathrm{a}$ ameliorates adipose tissue dysfunction. Mol Cell Biol 2013;33:904-17.

31. Elias I, Franckhauser S, Ferré T, Vilà L, Tafuro S, Muñoz S, et al. Adipose tissue overexpression of vascular endothelial growth factor protects against diet-induced obesity and insulin resistance. Diabetes 2012;61:1801-13.

32. Bråkenhielm E, Veitonmäki N, Cao R, Kihara S, Matsuzawa $Y$, Zhivotovsky $B$, et al. Adiponectin-induced antiangiogenesis and antitumor activity involve caspasemediated endothelial cell apoptosis. Proc Natl Acad Sci U S A 2004;101:2476-81.

33. Malavazos AE, Cereda E, Ermetici F, Caccialanza R, Briganti S, Rondanelli $M$, et al. The "lipid accumulation product" is associated with 2-hour postload glucose outcomes in overweight/obese subjects with nondiabetic fasting glucose. Int J Endocrinol 2015;2015:836941. 